\title{
VEGF-Targeting Strategies against Diabetic Nephropathy: Obsolete or Still Promising?
}

\author{
Katsuyuki Tanabe* and Jun Wada \\ Department of Nephrology, Rheumatology, Endocrinology and Metabolism, Okayama University, Japan \\ Received: January 30, 2018; Published: February 13, 2018
}

*Corresponding author: Katsuyuki Tanabe, Department of Nephrology, Rheumatology, Endocrinology and Metabolism, Okayama University Graduate School of Medicine, Dentistry and Pharmaceutical Sciences, 2-5-1 Shikata-cho, Kita-ku, Okayama 700-8558, Japan, Tel: 81-86-235-7235;

Email: tanabek@okayama-u.ac.jp

Abstract

Vascular endothelial growth factor (VEGF)-A is well known to be involved in the pathogenesis of diabetic nephropathy. Although it may be a promising therapeutic target, recent evidence that anti-VEGF therapy induces renal thrombotic micro-angiopathy has tempered interest in its use. We discuss the necessary conditions for future VEGF-targeting therapy against diabetic nephropathy.

Keywords: Diabetic nephropathy; VEGF-A; Endothelial dysfunction; Podocytes

\section{Introduction}

Diabetic nephropathy has become a leading cause of end-stage kidney disease in developed countries. Moreover, the worldwide outbreak of obesity and diabetes would result in a dramatic increase of diabetic nephropathy in developing countries such as those in Asia and Latin America. Many researchers continue to develop novel therapeutic strategies against it. The involvement of vascular endothelial growth factor (VEGF)-A has been well recognized in the pathogenesis of diabetic nephropathy. Several previous studies reported that inhibition of VEGF-A successfully prevented albuminuria and glomerular pathology in diabetic animal models [1].

Although VEGF-A is a promising therapeutic target for diabetic nephropathy, the enthusiasm for VEGF-targeting therapy was tempered by clinical evidence that bevacizumab, a monoclonal antiVEGF-A antibody, caused proteinuria and renal thrombotic microangiopathy in cancer patients [1,2]. In this review, we will describe the association between VEGF- A expression and the progression of diabetic nephropathy, and then, discuss the necessary conditions for future VEGF-targeting therapy against the disease.

\section{Increased VEGF-A Induces Early Stage Diabetic Nephrop- athy}

In glomeruli, VEGF-A is mainly expressed in podocytes. It acts on glomerular endothelial cells to maintain their structure and function [3], and possibly on mesangial cells to regulate the expression of mesangial matrix proteins [4]. In diabetes, hyperglycemia induces up regulation of VEGF- A in podocytes at least in the early stage, accompanied by micro-albuminuria and mesangial expansion. Given the previous reports that podocyte-specific VEGF-A over expression or systemic VEGF receptor-2 activation in mice caused albuminuria and mesangial expansion [5,6], increased expression of VEGF-A may be sufficient to induce the findings observed in early stage of diabetic nephropathy (Figure 1A). Indeed, anti-VEGF antibody was shown to ameliorate albuminuria and glomerular alterations in diabetic mice [7].

\section{Endothelial Dysfunction is Necessary for Advanced Stage Diabetic Nephropathy}

Increased VEGF-A alone is insufficient to induce advanced stage diabetic nephropathy, characterized by massive proteinuria, as well as nodular lesions in glomeruli. Despite the difficulty of inducing advanced glomerular lesions in wild-type mice, diabetes successfully resulted in the formation of nodular lesions in podocyte-specific VEGF- A over expressing mice [8]. Thus, some diabetes-induced aggravating factors, in addition to VEGF-A, should be required for the progression to advanced stage.

The most important factor among these is probably endothelial dysfunction, considering the evidence that diabetic endothelial nitric oxide synthase (eNOS, also known as NOS3) knockout mice developed massive proteinuria and advanced glomerular lesions, including nodular sclerosis and mesangiolysis [9-11]. Furthermore, podocyte-specific VEGF-A over expression in NOS3 knockout mice was reported to induce nodular glomerulosclerosis even without diabetes [12]. Taken together, diabetes-induced endothelial 
dysfunction, combined with increased VEGF-A, may accelerate albuminuria and glomerular alterations from early to advanced stage (Figure 1B).

\section{VEHG-A Expression Decreases with the Disease Progres-} sion

Increased expression of VEGF-A is observed in early stage diabetic nephropathy. However, as the disease progresses, VEGF-A is depleted in glomeruli [13]. This is likely to be due to loss of podocytes, the source of VEGF-A. Since podocyte-specific VEGF-A knockout mice were demonstrated to develop renal thrombotic micro-angiopathy, as observed in patients treated with anti-VEGF antibody [2], decreased expression of VEGF- A in glomeruli could promote endothelial injury. In addition, diabetes in podocytespecific VEGF-A knockout mice led to the prominent glomerular scarring [14].Therefore, an excessive reduction of VEGF-A would result in the devastating glomerular changes seen in diabetes (Figure 1C).

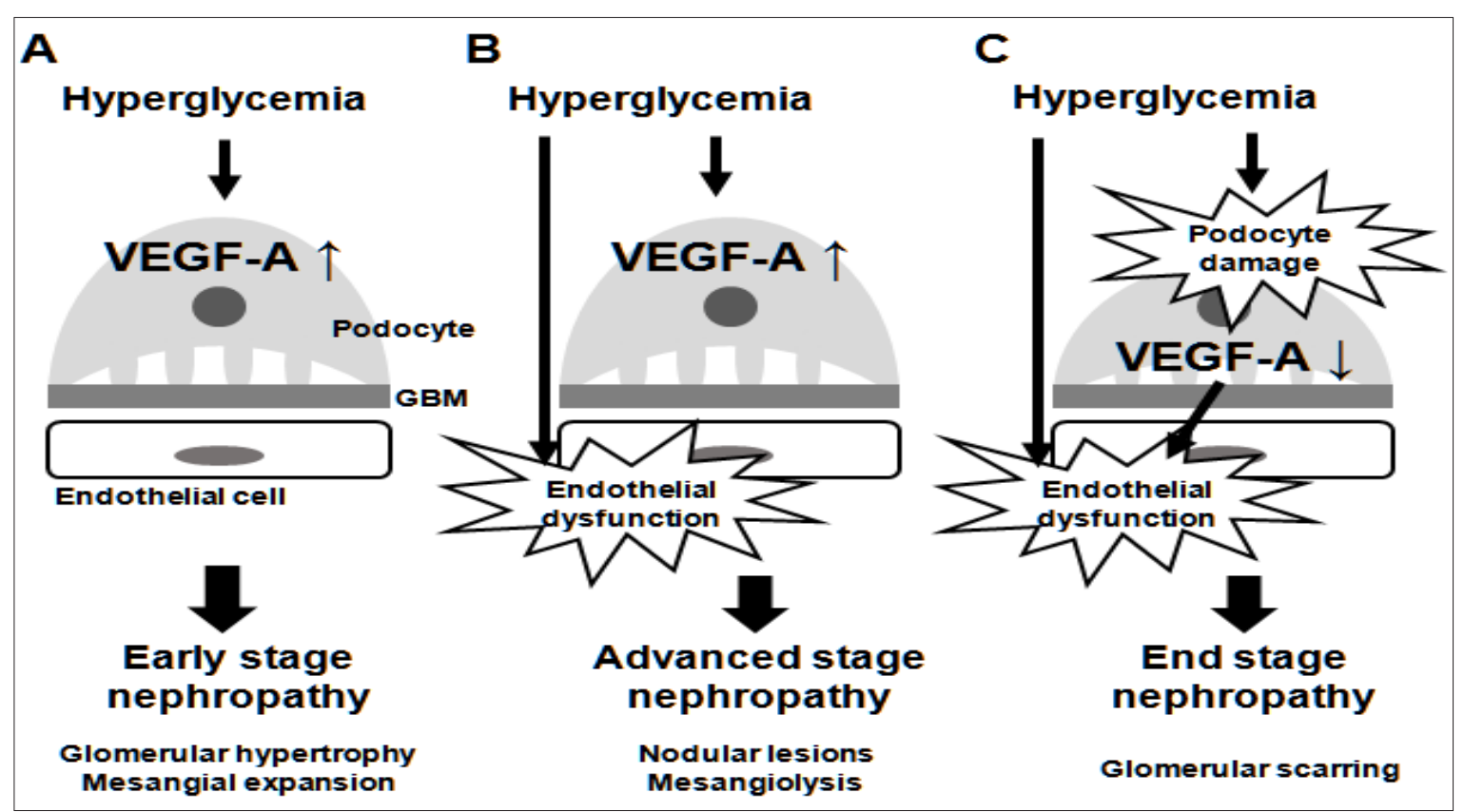

Figure 1: Vascular endothelial growth factor (VEGF-A) expression and the stages of diabetic nephropathy. VEGF-A in glomeruli is mainly expressed in podocytes. In the early stage (A), diabetes-induced increase in VEGF-A expression causes mesangial expansion. However, in the advanced stage (B), increased VEGF-A alone is insufficient to induce nodular glomerulosclerosis and mesangiolysis. Endothelial dysfunction, combined with increased VEGF-A, is required for the development of such characteristics. As glomerular injury progresses, VEGF-A expression could be decreased through podocyte damage (C). Depletion of VEGF-A in diabetes results in glomerular scarring. GBM = glomerular basement membrane.

What are the necessary conditions for Future VEGFTargeting Therapy?

Since anti-VEGF therapy for cancer could induce renal glomerular endothelial injury, enthusiasm for VEGF-targeting strategies against diabetic nephropathy has been dampened. However, VEGF-targeting therapy may still have beneficial potential, if it could meet some conditions. Based on the association between VEGF-A expression and the progression of diabetic nephropathy described above, we propose two necessary conditions for VEGFtargeting strategies against diabetic nephropathy.

A. VEGF-targeting therapy should not cause excessive reduction of VEGF-A.

B. VEGF-targeting therapy should not promote endothelial dysfunction.

Anti-VEGF antibody and soluble fms-like tyrosine kinase 1 (Flt1) directly bind to and deplete VEGF-A. Despite their beneficial effects in diabetic animal models [7,15], these agents accelerate endothelial injury, leading to glomerulosclerosis in the long term, as shown in (Figure 1C). Therefore, these are excluded from candidate therapeutic agents for diabetic nephropathy. Endostatin, a collagen-derived anti-angiogenic agent, was also shown to have renoprotective effects in diabetic mice [16]. This agent could interfere with VEGF signaling, but does not induce excessive reduction of VEGF-A. However, unfortunately, almost all antiangiogenic agents including endostatin are demonstrated to have pro-apoptotic effects on endothelial cells in vitro [1]. Therefore, anti-angiogenic agents intrinsically have the potential risk to cause endothelial dysfunction. Such important concerns have to be solved to develop novel VEGF-targeting therapies against diabetic nephropathy in future.

We previously reported the protective roles of vasohibin-1 (VASH1), an endothelium-derived negative feedback regulator of VEGF-induced angiogenesis, in a diabetic nephropathy mice model [17-19]. As VASH1 promotes endothelial survival in contrast to other anti-angiogenic agents [20], we consider it as a promising candidate for future VEGF-targeting therapy against diabetic nephropathy. However, the detailed mechanism by which VASH1 
inhibits VEGF signaling remains to be clarified. Recently, vasohibin-2 (VASH2), a homologue of VASH1, was also implicated with diabetic nephropathy [21], but whether VASH2 is a novel target for diabetic nephropathy remains unknown at present.

\section{Conclusion}

As mentioned above, VEGF-targeting therapy against diabetic nephropathy has to avoid excessive reduction of VEGF- A as well as endothelial dysfunction. If such a strategy meets the necessary conditions, it still has beneficial potential. If not, it would be obsolete.

\section{References}

1. Tanabe K, Maeshima Y, Sato Y, Wada J (2017) Antiangiogenic Therapy for Diabetic Nephropathy. BioMed research international p. 12.

2. Eremina V, Jefferson JA, Kowalewska J, Hochster H, Haas M, et al. (2008) VEGF inhibition and renal thrombotic microangiopathy. The New England journal of medicine 358(11): 1129-1136.

3. Sison K, Eremina V, Baelde H, Min W, Hirashima M, et al. (2010) Glomerular structure and function require paracrine, not autocrine, VEGF-VEGFR-2 signaling. Journal of the American Society of Nephrology: JASN 21(10): 1691-1701.

4. Amemiya T, Sasamura H, Mifune M, Kitamura Y, Hirahashi J, et al. (1999) Vascular endothelial growth factor activates MAP kinase and enhances collagen synthesis in human mesangial cells. Kidney international 56(6): 2055-2063.

5. Veron D, Reidy KJ, Bertuccio C, Teichman J, Villegas G, et al. (2010) Overexpression of VEGF- A in podocytes of adult mice causes glomerular disease. Kidney international 77(11): 989-999.

6. Sato W, Tanabe K, Kosugi T, Hudkins K, Lanaspa MA, et al. (2011) Selective stimulation of VEGFR2 accelerates progressive renal disease. The American journal of pathology 179(1): 155-166.

7. de Vriese AS, Tilton RG, Elger M, Stephan CC, Kriz W, et al. (2001) Antibodies against vascular endothelial growth factor improve early renal dysfunction in experimental diabetes. Journal of the American Society of Nephrology: JASN 12(5): 993-1000.

8. Veron D, Bertuccio CA, Marlier A, Reidy K, Garcia AM, et al. (2011) Podocyte vascular endothelial growth factor (Vegf(1)(6)(4)) overexpression causes severe nodular glomerulosclerosis in a mouse model of type 1 diabetes. Diabetologia 54(5): 1227-1241.

9. Nakagawa T, Sato W, Glushakova O, Heinig M, Clarke T, et al. (2007) Diabetic endothelial nitric oxide synthase knockout mice develop advanced diabetic nephropathy. Journal of the American Society of Nephrology: JASN 18(2): 539-550.
10. Tanabe K, Lanaspa MA, Kitagawa W, Rivard CJ, Miyazaki M, et al. (2012) Nicorandil as a novel therapy for advanced diabetic nephropathy in the eNOS-deficient mouse. American journal of physiology Renal physiology 302(9): F1151-F1160.

11. Nakagawa T, Tanabe K, Croker BP, Johnson RJ, Grant MB, et al. (2011) Endothelial dysfunction as a potential contributor in diabetic nephropathy. Nature reviews Nephrology 7(1): 36-44.

12. Veron D, Aggarwal PK, Velazquez H, Kashgarian M, Moeckel G, et al. (2014) Podocyte-specific VEGF-a gain of function induces nodular glomerulosclerosis in eNOS null mice. Journal of the American Society of Nephrology: JASN 25(8): 1814-1824.

13. Baelde HJ, Eikmans M, Doran PP, Lappin DW, de Heer E, et al. (2004) Gene expression profiling in glomeruli from human kidneys with diabetic nephropathy. American journal of kidney diseases: the official journal of the National Kidney Foundation 43(4): 636-650.

14. Sivaskandarajah GA, Jeansson M, Maezawa Y, Eremina V, Baelde HJ, et al. (2012) Vegfa protects the glomerular microvasculature in diabetes. Diabetes 61(11): 2958-2966.

15. Ku CH, White KE, Dei Cas A, Hayward A, Webster Z, et al. (2008) Inducible overexpression of sFlt-1 in podocytes ameliorates glomerulopathy in diabetic mice. Diabetes 57(10): 2824-2833.

16. Ichinose K, Maeshima Y, Yamamoto Y, Kitayama H, Takazawa Y, et al. (2005) Antiangiogenic endostatin peptide ameliorates renal alterations in the early stage of a type 1 diabetic nephropathy model. Diabetes 54(10): 2891-2903.

17. Nasu T, Maeshima Y, Kinomura M, Hirokoshi-Kawahara K, Tanabe K, et al. (2009) Vasohibin-1, a negative feedback regulator of angiogenesis, ameliorates renal alterations in a mouse model of diabetic nephropathy. Diabetes 58(10): 2365-2375.

18. Hinamoto N, Maeshima Y, Yamasaki H, Nasu T, Saito D, et al. (2014) Exacerbation of diabetic renal alterations in mice lacking vasohibin-1. PloS one 9(9): e107934.

19. Saito D, Maeshima Y, Nasu T, Yamasaki H, Tanabe K, et al. (2011) Amelioration of renal alterations in obese type 2 diabetic mice by vasohibin-1, a negative feedback regulator of angiogenesis. American journal of physiology Renal physiology 300(4): F873-F886.

20. Miyashita H, Watanabe T, Hayashi H, Suzuki Y, Nakamura T, Ito S, et al. (2012) Angiogenesis inhibitor vasohibin-1 enhances stress resistance of endothelial cells via induction of SOD2 and SIRT1. PloS one 7(10): e46459.

21. Arata Y, Tanabe K, Hinamoto N, Yamasaki H, Sugiyama H, et al. (2017) Immunohistochemistry of Vasohibin-2 in Human Kidney Disease : Implications in Impaired Glucose Tolerance and Reduced Renal Function. Acta medica Okayama 71(5): 369-380.

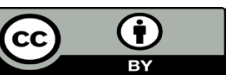

This work is licensed under Creative Commons Attribution 4.0 License

Submission Link: http://biomedres.us/submit-manuscript.php

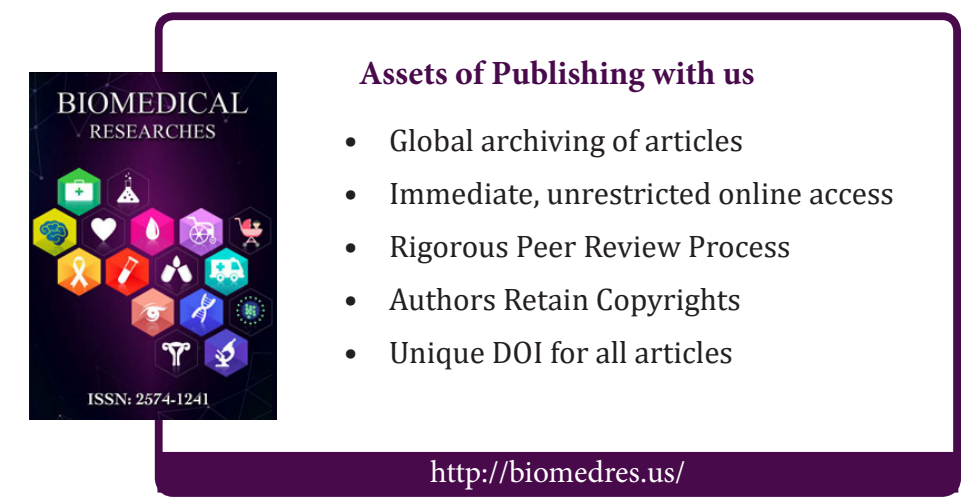

